\title{
UWB LOCALIZATION OF NODES FOR SECURING A MANET
}

\author{
Mr.C.Susil Kumar ${ }^{1}$, M.Ashra ${ }^{2}$, P.M.Jessintha ${ }^{3}$, P.S.Kavitha ${ }^{4}$, \\ ${ }^{1}$ Assistant Professor, ${ }^{2,3,4}$ U.G Student \\ Department of Electronics and Communication Engineering, \\ Velammal College of Engineering and Technology, Madurai, Tamilnadu, India.
}

\begin{abstract}
Localization of nodes in an infrastructure less network serves many purposes. Several issues relating to security, routing, etc it can be solved if only the actual location of nodes were known. Existing approaches estimate the location of a node in a network by using received signal strength indicator (RSSI), Time of Arrival, Time difference of Arrival and, if directional antennas are available, Direction of Arrival. In these methods the localization accuracy is less (in the order of 20cm). The aim of this paper is to localize nodes in adhoc networks with improved accuracy using ultra wide band.The proposed method uses a train of low amplitude pulses of high bandwidth, which reduces the energy consumption, effects due to small scale fading, and dispersion in time and frequency. The network was simulated in NS-2 with UWB extension and the localization accuracy was found to be improved (upto $1 \mathrm{~cm}$ ).
\end{abstract}

\section{KEYWORDS}

Localization, MANET, UWB, TDOA

\section{INTRODUCTION}

An ad hoc network is a collection of wireless mobile nodes dynamically forming a temporary network without the use of existing network infra-structure or centralized administration. Due to the limited transmission range of wireless network interfaces, multiple network hops may be needed for one node to exchange data with another across the network. Localization is defined as the phenomenon of obtaining location information of nodes whose positions are not known. Thus, in the context of MANETs, localization means finding location of mobile nodes (MNs). Location awareness is an essential feature to gain full benefits of ubiquitous computing. Thus, several localization algorithms are introduced to estimate location of MNs. Location information obtained from localization algorithms can be useful for accessing various location based services such as inventory tracking, habitat monitoring, rescue operations, museum tour guide, etc. The position of mobile station (MS)can be determined using multiple radio signals transmitted or received by the MS. Some location estimation methods like assisted global positioning system (A-GPS) are based on signals transmitted from satellites. So far , a wide variety of network-based positioning techniques have been proposed which use measurements obtained within the cellular networks, such as received signal strength (RSS),time of arrival (TOA),time difference of arrival (TDOA), and angle of arrival (AOA) methods. UWB technology provides an excellent means for wireless positioning due to its high resolution capability in the time domain. Its ability to resolve multipath components makes it possible to obtain accurate location estimates without the need for complex estimation algorithms. Although the fundamental mechanisms for localization, including 
AOA, TOA, TDOA and SS-based methods, apply to all radio air interfaces, some positioning techniques are favoured by UWB-based systems using ultra wide bandwidths.

\subsection{RELATED WORKS}

Localization in mobile ad hoc network is an active area of research and so there are some existing literature surveys on this topic .But there are some existing techniques which use to localize in ad hoc networks RSSI,ToA,AoA,TDoA.The RSSI techniques uses high computation cost, database maintenance is complex, and it is less accurate .In order to overcome this, UWB localization is proposed. This literature gives comprehensive summary of these techniques along with the existing localization schemes. Since ad hoc network is an infra structure less network there is no confidentiality while transmitting the packets.

\subsection{LOCALIZATION IN INFRASTRUCTURE-LESS NETWORKS}

In self-organized wireless networks such as ad hoc or sensor networks, most nodes have the same transmission range. At the same time, most nodes can establish the trust relationship with only their direct neighbours through interactions. Therefore, we cannot locate a group of special nodes that can serve as senders to cover the whole network. Fortunately, the self-organization property allows the wireless less nodes to help each other. The nodes already learning their position can serve as anchor node for other devices. Under this condition we need to investigate the required density and distribution of the initial anchor nodes and wireless devices .so that the localizations procedure can propagate throughout the network. An example scenario is shown in Fig.1.We assume that all nodes in the self -organized network have the same transmission distance. To initialize the localization distance, we deploy a small group of anchor nodes that also have the same transmission disatance in the network.

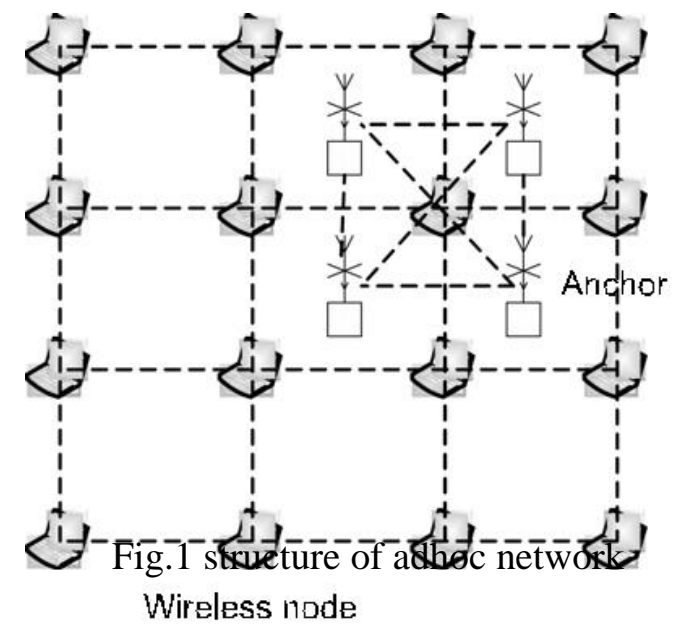

\section{EXISTING METHODS}

There are several existing methods for finding the location of nodes in ad hoc networks. They are Received signal strength indicator (RSSI), time based methods (ToA, TDoA), Angle of Arrival (AoA), hyperbolic trilateration, triangulation, Maximum likelihood estimation (ML). 
RSSI measures the power of the signal and calculate the propagation loss based on the known transmitted power. This method uses only RF signals which is relatively a cheap solution for localization. RSSI does not use any extra devices because all mobile are likely to have radios. The performance is not good as other, due to multipath path fading.

\subsection{TIME BASED METHODS(TOA,TDOA)}

These methods record the sending time of a packet and receiving time of a packet. Using the recorded time, time difference between the nodes are calculated. The propogation time can be directly related to the distance with the help of the propagation speed. These methods can be applied to various signals like RF, acoustic, IR and Ultra sound. TDoA methods are accurate under line-of-sight conditions. But LoSconditions is difficult to achieve .Acoustic signals also show multipath propagation effects that may reduce the accuracy of localization.

\section{ANGLE-OF-ARRIVAL}

AoA determines the angle at which signals are received and use geomentric relationship to find the node positions measurement is a method for determining the direction of propagation of a audio-frequency wave incident on an antenna array. AoA determines the direction by measuring the Time Difference of Arrival (TDoA) at individual elements of the array from these delays the AoA can be calculated the gain of the antenna array. In AoA, the delay of arrival at each element is measured directly and converted to an AoA measurement.

\subsection{TRIANGULATION}

Triangulation is the process of determining the location of a point by measuring angles to it from known points at either end of a fixed baseline, rather than measuring distances to the point directly (trilateration). The point can then be fixed as the third point of a triangle with one known side and two known angles.Fig.2.shown below

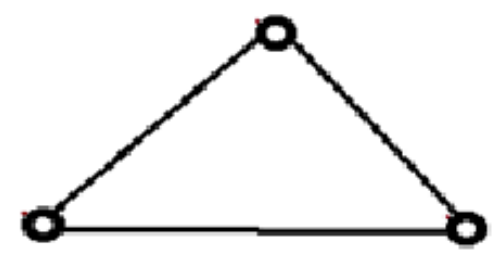

TRIANGULATION

Fig.2.Triangulation

\section{PROPOSED METHOD}

The proposed method is to localize the node in mobile adhoc network with improved accuracy using ultra wide band. In our proposed method a train of low amplitude pulses is sent so that it reduces the energy consumption. Consider the anchor nodes and mobile nodes as shown in Fig.4. The anchor nodes are represented by node 0 , node 1 and node 2 . The mobile nodes whose locations are to be estimated are represented by node 3, node 4, and node5.The Fig.3. Shown below. 


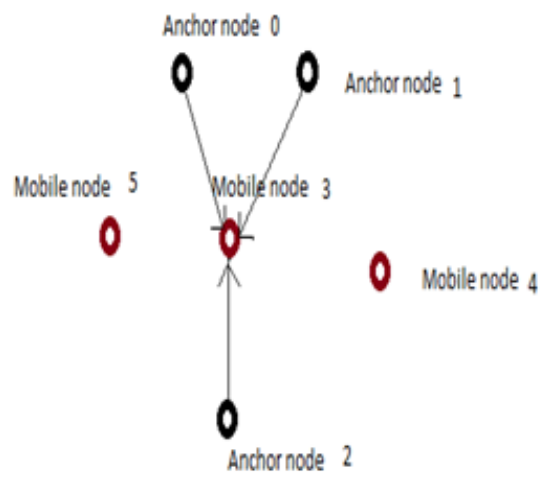

Fig.3.

Fig.3.Node formation

The anchor nodes send packets to un-localized mobile nodes. Here, co-ordinates of anchor nodes are already known. By using Time Difference of arrival (TDoA) techniques, the difference between sending and receiving time of packets from anchor nodes to mobile nodes can be calculated. Accuracy will be higher since UWB has excellent immunity time and frequency dispersion. UWB also offers a large bandwidth that improves reliability, as the signal contains different frequency components, offers high resolution radars with improved ranging accuracy.

\subsection{TRILATERATION METHOD}

After finding the distance between anchor nodes and mobile nodes the distance is used as radius to draw the circle, by keeping the mobile node as the center point. Thus, Fig.4.shown below

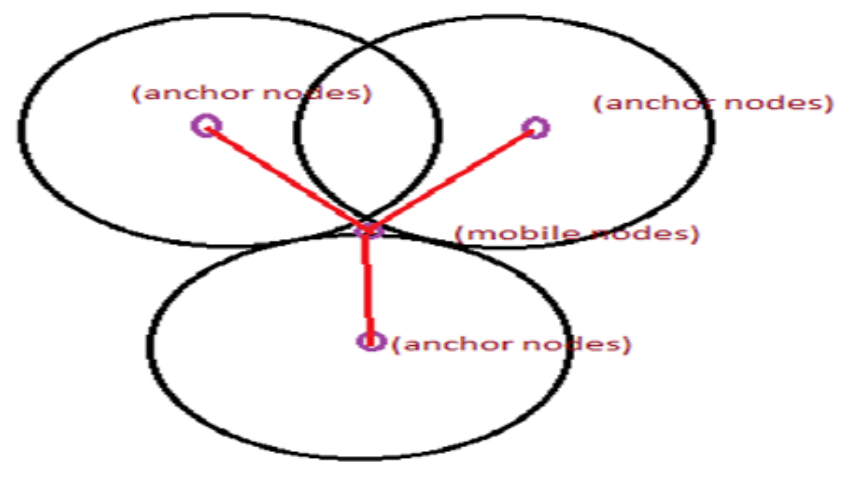

Fig.4.Trilateration method

By using the following trace files given below (Fig.5.),we can estimate the co-ordinates of X,Y,Z with the technique of TDoA (i.e) Calculate the time difference between sending and receiving signal ,by substituting the values obtained .Thus, the value of distance (d) will be known, hence by using the given co-ordinates for already known anchor nodes and calculated distance (d) we can estimate the unknown co-ordinates of X,Y,Z.It may consists of size of the packets, protocols used ,result of acknowledgement, agent file ,ID sequence number allocated while transmitting the packets to particular nodes to another. 


\section{2) TRACEFILE}

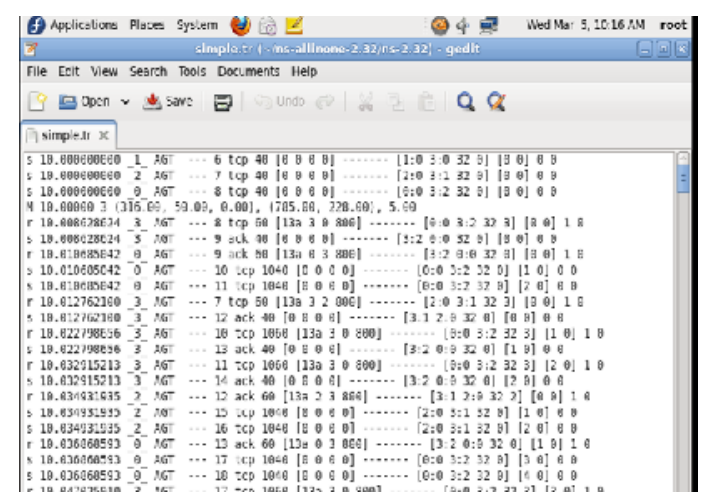

Fig.5.Trace file

\subsection{TRILATERATION FORMULA TO DERIVE THE CO-ODRINATES OF MOBILE NODES}

$$
\begin{gathered}
\mathrm{X}=\frac{r_{1}^{2}-r_{2}^{2}+\left(x_{2}-x_{1}\right)^{2}+\left(y_{2}-y_{1}\right)^{2}+\left(z_{2}-z_{1}\right)^{2}}{2 \sqrt{\left(x_{2}-x_{1}\right)^{2}+\left(y_{2}-y_{1}\right)^{2}+\left(z_{2}-z_{1}\right)^{2}}} \\
\mathrm{Y}=\frac{r_{1}^{2}-r_{3}^{2}-x^{2}+x_{3}^{2}+\left(y-y_{3}\right)^{2}}{2\left(y-y_{3}\right)} \\
\mathrm{Z}= \pm \sqrt{r_{1}^{2}-x^{2}-y^{2}}
\end{gathered}
$$

\section{RESULT AND DISCUSSION}

\subsection{SIMULATION}

In Fig.6. it shows the result of our proposed method which consist of mobile nodes (50 nodes). Here ,the red color nodes indicates the anchor nodes and green indicates the unlocalized nodes, by using TDoA techniques it can be localized by estimating the known co-ordinates of anchor nodes.

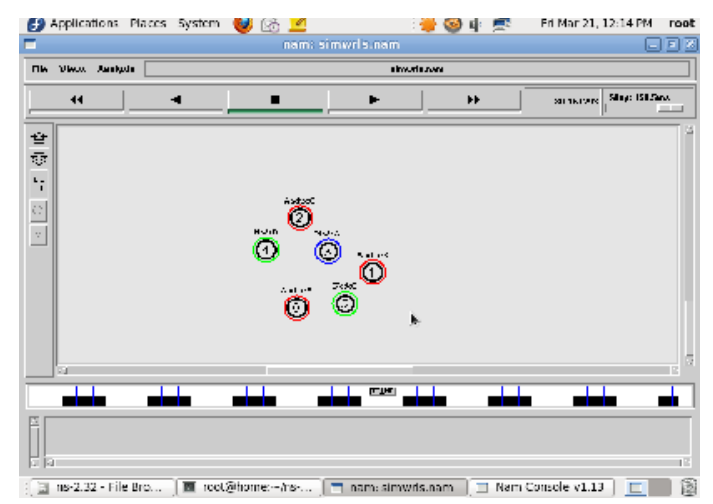


Fig.6.simulation output

Here, we are using NS2 with UWB extension software to simulate the unlocalized mobile nodes ,which has been aldready shown in Fig.6. The unlocalized nodes can be localized by estimating the the coordinates of anchor nodes, as we implies earlier the coordinates of anchor nodes should be known. Localization of stationary nodes and localization of mobile nodes were calculated and tabulated below. The known coordinates of anchor nodes X,Y,Z were randomly selected and calculated the approximate location of stationary and mobile nodes.

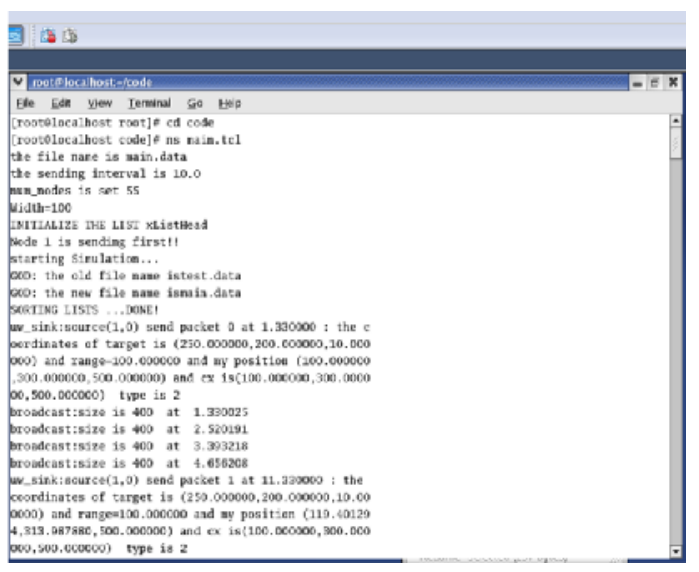

Fig.7.localization output

Thus, Fig.7. Shows the simulated result of location for unlocalized mobile nodes. The coordinates of $\mathrm{X}, \mathrm{Y}, \mathrm{Z}$ can be achieved. Let us compare the efficiency of given existing methods with our proposed method. Since we are using a train of very low pulse amplitude over a larger bandwidth our accuracy level is improved to $1 \mathrm{~cm}$.

\subsection{WITHOUT UWB EXTENSION}

\section{2 .1 LOCALIZATION OF STATIONARY NODES:}

$\mathrm{X}=316.0, \mathrm{Y}=50.0, \mathrm{Z}=0$

\begin{tabular}{|c|c|c|}
\hline $\mathrm{X}$ & $\mathrm{Y}$ & $\mathrm{Z}$ \\
\hline 320.02 & 53.5 & -1.09 \\
\hline 318.17 & 54.24 & 0.96 \\
\hline 310.91 & 51.07 & 1.21 \\
\hline 321.22 & 52.62 & -0.81 \\
\hline 319.57 & 53.80 & 1.12 \\
\hline
\end{tabular}




\subsubsection{LOCALIZATION OF MOBILE NODES}

\begin{tabular}{|c|c|c|c|c|c|}
\hline \multicolumn{3}{|c|}{ Actual } & \multicolumn{3}{c|}{ Calculated } \\
\hline $\mathrm{X}$ & $\mathrm{Y}$ & $\mathrm{Z}$ & $\mathrm{X}$ & $\mathrm{Y}$ & $\mathrm{Z}$ \\
& & & & & \\
\hline 315.0 & 50.0 & 0.0 & 312.12 & 52.7 & 0.54 \\
\hline 320.1 & 52.5 & 0.0 & 316.04 & 54.5 & -0.32 \\
\hline 324.9 & 54.9 & 0.0 & 321.53 & 51.3 & 1.04 \\
\hline 330.2 & 57.6 & 0.0 & 327.6 & 59.9 & 0.77 \\
\hline 335 & 60 & 0.0 & 331.3 & 62 & 1.02 \\
\hline
\end{tabular}

\subsection{WITH UWB EXTENSION}

\subsubsection{LOCALIZATION OF STATIONARY NODES}

$\mathrm{X}=316.0, \mathrm{Y}=50.0, \mathrm{Z}=0$

\begin{tabular}{|c|c|c|}
\hline $\mathrm{X}$ & $\mathrm{Y}$ & $\mathrm{Z}$ \\
\hline 316.02 & 50.7 & -0.92 \\
\hline 315.21 & 51.42 & 0.62 \\
\hline 315.09 & 51.07 & 0.11 \\
\hline 316.43 & 49.76 & -0.21 \\
\hline 317.05 & 50.82 & 0.72 \\
\hline
\end{tabular}




\section{3 .2 LOCALIZATION OF MOBILE NODES}

\begin{tabular}{|c|c|c|c|c|c|}
\hline \multicolumn{3}{|c|}{ Actual } & \multicolumn{3}{c|}{ Calculated } \\
\hline $\mathrm{X}$ & $\mathrm{Y}$ & $\mathrm{Z}$ & $\mathrm{X}$ & $\mathrm{Y}$ & $\mathrm{Z}$ \\
\hline 315.0 & 50.0 & 0.0 & 315.02 & 50.7 & 0.47 \\
\hline 320.1 & 52.5 & 0.0 & 321.4 & 51.2 & -0.3 \\
\hline 324.9 & 54.9 & 0.0 & 323.3 & 53.4 & 0.04 \\
\hline 330.2 & 57.6 & 0.0 & 329.8 & 58.2 & 0.7 \\
\hline 335 & 60 & 0.0 & 333.7 & 59 & 0.87 \\
\hline
\end{tabular}

\subsection{PERCENTAGE (\%) ERROR}

\begin{tabular}{|c|c|c|c|}
\hline \multicolumn{2}{|c|}{ Without uwb (\%) } & \multicolumn{2}{|c|}{ With uwb (\%) } \\
\hline & 0.91 & & 0.63 \\
\hline & 1.26 & & 0.40 \\
\hline & 1.03 & & 0.49 \\
\hline & 0.78 & & 0.12 \\
\hline & 1.10 & & 0.38 \\
\hline Avg & 1.016 & Avg & 0.404 \\
\hline
\end{tabular}

\section{RESULTS AND DISCUSSION}

We assumed two cases to localize the mobile nodes. They are

1) Localization of stationary nodes.

2) Localization of mobile nodes.

Thus, we calculated the localization of mobile nodes and localization of stationary nodes by using the actual coordinates of $\mathrm{X}, \mathrm{Y}, \mathrm{Z}$ for both with and without UWB extension. Then we estimated the percentage error (\%) for both the cases .Percentage error for UWB extension is less. It clearly 
shows the accuracy is improved by using UWB. Without UWB the localization error is more so it misleads the information sent by a particular mobile node. We have plot the change in percentage error (\%) versus distance from anchor nodes. Euclidean distance Vs \% error has been plotted and shown. Distance from anchor nodes are calculated by trilateration method

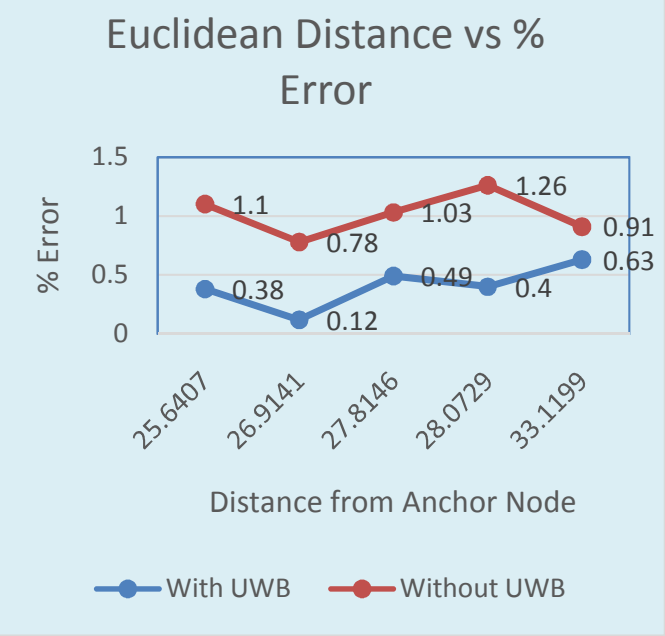

Fig.8. Euclidean distance vs \% error

Hence, Fig. 8 shows the improved accuracy with UWB is when compared to the without UWB .When the distance increases the error also increases it minimised by using uwb.

\section{REFERENCES}

[1] ZhiyongYang, KaideHuang ,XuemeiGuo,Guoli Wang . A real-time device-free localization system using correlated RSS measurements. EURASIP Journal on Wireless Communications and Networking, July 2013

[2] Musa Bora Zeytinci, Veli Sari, Frederic Kerem Harmanci^, Emin Anarim and Mehmet Akar*.Location estimation using RSS measurements with unknown path loss exponents. EURASIP Journal of wireless comm. \& networking. 2013

[3] SinanGezici, ZhiTian, Georgios B. Giannakis, Hisashi Kobayashi, Andreas F. Molisch, H. Vincent Poor, and ZaferSahinoglu, Localization via Ultra Wide Band Radios.

[4] Federico Penna1 and Danijela Cabric2,EURASIP Journal onWireless Communications and Networking,2013. Cooperative DoA-only localization of primary users in cognitive radio networks.

[5] Amitangshu pal, Localization algorithms in wireless sensor networks current approaches and future challenges,ISSN 1943-3581,july 2010.

[6] J. Yi, J. Koo, and H. Cha, "A localization technique for mobile sensor networks using archived anchor information," in Proc. IEEE Conf. Sensor, Mesh, and Ad Hoc Communications and Networks (SECON), California, USA, 2008, pp. 64-72.

[7] Michael TUCHLER, Volker SCHWARZ, Alexander HUBER, Accuracy of an UWB localization system based on a CMOS chip,WPNC'05.

[8] Mohammed Aquil Mirza1*,MuhammedZeeshan Shakir2 and Mohammed-slim Alouini3,A Scalable global positioning system-free localization scheme for underwater wireless sensor networks, 2013.

[9] Chi-Tung1, Shu-Yan Huang2* and luon -Chang Lin 2,3*, Perfect Forward Secrecy for locationaware wireless sensor networks,EURASIP journal on woreless communication and networking 2012.

[10] ZhiweiLi,WeichoWang UNC charlotte, NC 28223, US, Node localization through physical layer network coding : Bootstrap, security, and accuracy,5 april 2012.

[11] Poovendran R, Wang C, Roy S. Secure Localization and Time Synchronization for Wireless Sensor and Ad hoc Networks.New York Inc: Springer-Verlag; 2007. 